\title{
Psychedelics alter metaphysical beliefs
}

Christopher Timmermann ${ }^{1}$, Hannes Kettner ${ }^{1}$, Chris Letheby ${ }^{2,3}$, Leor Roseman ${ }^{1}$, Fernando E. Rosas ${ }^{1,4,5}$ and Robin L. Carhart-Harris ${ }^{1}$

${ }^{1}$ Centre for Psychedelic Research, Division of Psychiatry, Department of Brain Sciences, Imperial College London

${ }^{2}$ Department of Philosophy, University of Western Australia

${ }^{3}$ Department of Philosophy, University of Adelaide

${ }^{4}$ Data Science Institute, Imperial College London

${ }^{5}$ Centre for Complexity Science, Imperial College London

Corresponding Author: Christopher Timmermann

E-mail address: c.timmermann-slater15@imperial.ac.uk 


\begin{abstract}
Are psychedelics able to induce lasting changes in metaphysical beliefs? While it is popularly believed that they can, this has never been systematically tested. Here we exploited a large sample derived from prospective online surveying to determine whether and how beliefs concerning the nature of reality, consciousness, and freewill, change after psychedelic use. Results revealed significant shifts away from 'physicalist' or 'materialist' views, and towards panpsychism and fatalism, post use. These changes remained detectable at 6 months, and were associated with the extent of past use and improved mental-health outcomes. Path modelling suggested that the belief-shifts were moderated by impressionability at baseline and mediated by perceived emotional synchrony with others during the psychedelic experience. The observed belief-shifts post psychedelic use were confirmed by data from an independent controlled clinical trial. Together, these findings imply that psychedelic use has a causal influence on metaphysical beliefs - shifting them away from 'hard materialism'.
\end{abstract}




\section{Introduction}

Metaphysics is a branch of philosophy that studies themes such as the fundamental nature of reality, consciousness, and free will ${ }^{1}$. Research has shown that most of us hold distinct metaphysical positions - even though we may not be aware of $\mathrm{it}^{2-7}$. Metaphysical beliefs interface with such basic domains as health, religion, law, politics and education ${ }^{8-12}$. They are closely entwined with a society's culture and its stability ${ }^{13}$.

Although often held implicitly, metaphysical beliefs can become explicitly manifest during or after particularly intense life experiences or altered states ${ }^{14,15}$, such as near-death experiences ${ }^{16}$, meditation ${ }^{17}$, hypnosis ${ }^{18}$, experiences of 'awe'19, traumatic events $^{15,20}$, and psychedelic drug-induced experiences ${ }^{21-26}$.

Focusing specifically on psychedelics, recent evidence has demonstrated that psychedelics can reliably and robustly induce intense, profound, and personally meaningful experiences that have been referred to as 'mystical-type'27, 'spiritual' 28 , 'religious' ${ }^{29}$, 'existential'30, 'transformative ${ }^{31}$, or 'peak' ${ }^{32}$. It is tempting to hypothesize that these experiences operate as key mediators of potentially enduring transformations in metaphysical beliefs.

Some specific facets of these potentially transformative psychedelic experiences include: perceived encounters with 'supernatural' beings ${ }^{26,29}$, transcendence of the presumed physical bounds and laws of this 'consensus reality"23-26, encounters with an 'ultimate reality' ${ }^{\prime 2}$, witnessing or comprehending spatial and temporal vastness, a perception that the 'cosmos is fundamentally conscious' 25 and/or that all things are essentially inter-related or connected, i.e. the so-called 'unitive experience'33. 
The unitive experience is arguably the most tangible feature of these experiences $^{33,34}$. It is closely related to the so-called 'overview effect' 35 , 'universal insight' ${ }^{35}$, experience of 'awe'19,35,36 and so-called 'non-dual' states ${ }^{37}$. Such experiences appear to have a powerful capacity for mediating major shifts in perspective ${ }^{19,31,38}$, including shifts in metaphysical beliefs.

While some anecdotal, qualitative and retrospective reports hint that psychedelics can change metaphysical beliefs ${ }^{25,26,39}$, and that these shifts in beliefs are often explained post-hoc as having been triggered by revelations or insights ${ }^{40}$, there have been no formal, systematic, controlled and quantitative investigations of this phenomenon $^{41}$.

Addressing this knowledge gap, the present study sought to address three key questions:

1) Can psychedelics causally affect core beliefs concerning the nature of reality, consciousness and free will?

2) What is the relationship between the putative belief-changes and mental health?

3) What are the psychological mechanisms underlying the putative belief-shifts?

For this purpose, we developed a prospective survey requiring respondents answer questions pertaining to a range of metaphysical beliefs before and after attending a ceremonial retreat in which a psychedelic compound was taken. The external validity of these findings was subsequently examined via comparison with data derived from a randomized, controlled clinical trial in major depressive disorder, in which changes in beliefs were measured following psilocybin-therapy vs. a 6-week course of the selective-serotonin-reuptake-inhibitor, escitalopram. 


\section{Results}

\section{Non-physicalist Beliefs}

866 respondents completed baseline surveys enquiring about their metaphysical beliefs (see Supplementary Results and Supplementary Table 1 for sample characteristics). Items were formulated in a way to approximate classic metaphysical positions in non-specialist terms, and therefore do not necessarily measure precise philosophical positions, but rather folk beliefs associated with them. A factor analysis on the new belief items developed for the survey (together with 3 items derived from previous research ${ }^{6}$ ) revealed a single belief factor comprised of 9 items, which we have labelled Non-physicalist Beliefs (NPB; see Methods for factor analysis results). This single factor showed good internal consistency (Cronbach's alpha $=0.86)$, and included positive loadings (>.4) for items related to beliefs in separate and supernatural realms of existence, a non-natural unifying principle in reality, panpsychism, dualism, and solipsism/idealism. Items that loaded negatively $(<-0.4)$ referred to 'hard' monistic materialist or physicalist positions, or a belief in natural (as opposed to super-natural) explanations for phenomena in the universe (naturalism). These items and their loadings are shown in Table 1 (See Supplementary Table 2 for the full Metaphysical Beliefs Questionnaire). 
Table 1. Final items and factor loadings corresponding to the Non-physicalist Beliefs factor

Item

Factor

loading

There exists another separate realm or dimension beyond this physical world that can be experienced and visited. (Ontological

transcendentalism)

Visiting such immersive "realms" or "worlds" can sometimes depend on a supernatural / magical transition process or event. (Supernatural transcendentalism)

The universe obeys a unifying principle which is beyond any possible material or scientific explanation. (Non-naturalism)

There is just one primary reality: the physical; the mind (and/or consciousness) is just physical/functional properties of the brain and has an entirely material explanation. (Materialism)

Mind, consciousness, or soul is a fundamental quality of all things in the universe, either animate or inanimate. (Panpsychism)

The universe obeys a unifying principle which is (in theory) completely addressable by a material or scientific explanation. (Naturalism)

There are other realms of existence which are more important than everyday reality. (Primacy of other realms)

There are two separate realms of existence, the physical (body, brain and external world) and the mind, the latter being non-physical/non-material. (Dualism)

The physical world is an illusion generated by consciousness or the mind (Solipsism/ldealism)

We compared NPB scores before attending a retreat involving psychedelic use (baseline) with NPB scores 4 weeks and 6 months after the retreat. Pooling scores for the NPB factor, analyses revealed a significant shift away from physicalism at 4 weeks compared with baseline $(t(121)=3.66, p=0.001, d=0.33$, confidence interval, $95 \% \mathrm{Cl}=[0.12,0.39])$. These changes were sustained 6 months after the retreat $(t(121)=5.07],, p<0.0001, d=0.46,95 \% \mathrm{Cl}=[0.22,0.50])$ (Figure 1a). Larger effect sizes were found for respondents who were embarking on their first psychedelic experience (the so-called 'psychedelic naïve'), with significant changes 
found at 4 weeks $(t(52)=3.85, p=0.001, d=0.53,95 \% \mathrm{Cl}=[0.21,0.66])$ and 6 months $(t(52)=5.32, p<0.0001, d=0.73,95 \% \mathrm{Cl}=[0.36,0.80])$ (Supplementary Figure 1a). Analyses of each individual item for the NPB factor revealed increases in notions of transcendentalism, mind-body dualism, and panpsychism - among others, with some changes remaining significant for 6 months (see Figure 1b-left and Supplementary Figure $1 \mathrm{~b}$ for findings for 'naïve' respondents). Additionally, a significant positive correlation was found between previous psychedelic use and shifts away from the hard-materialism pole of the hard-materialism vs. hard-dualism spectrum (Figure 1b-right) at baseline $(r=0.223, p<0.0001)$. 
a
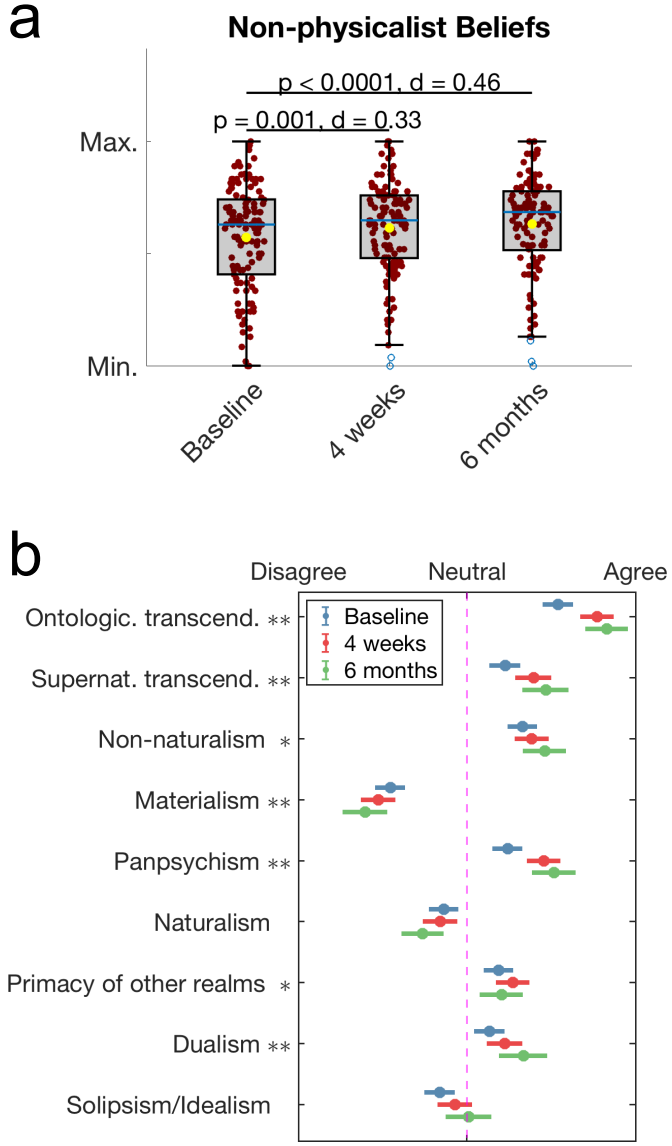
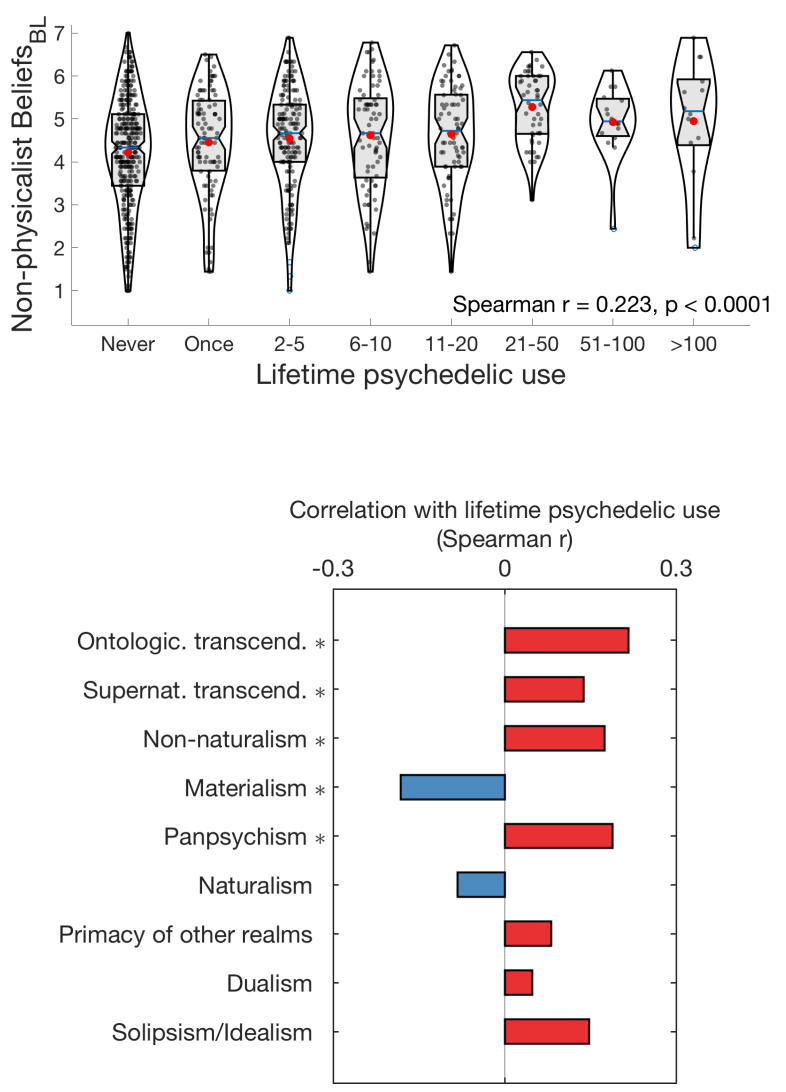

Figure 1. Psychedelic use is associated with shifts in metaphysical beliefs. Attending a psychedelic retreat was associated with shifts away from hard-materialistic views (a-left), and items associated with transcendentalism, non-naturalism, panpsychism, primacy of other realms, dualism and solipsism/idealism (b-left), with some changes enduring up to 6 months (Bonferroni-corrected). Additionally, significant positive relationships were observed between lifetime psychedelic use and baseline scores on metaphysical beliefs (a-right), and items referring to transcendentalism, non-naturalism, and panpsychism, while a negative relationship was found with materialism (b-right). (b-left: mean values and standard errors displayed $^{*}=$ Significant change at 4 weeks; ${ }^{* *}=$ Significant change at 6 months, Bonferronicorrected; B-right: ${ }^{*} p<0.0001$, Bonferroni-corrected).

\section{Fatalism}

Analysis of the prospective data revealed that the psychedelic retreat was associated with increases in scores of Fatalistic determinism ${ }^{4}$ (see Supplementary Methods for the items used) at 4 weeks versus baseline $(t(121)=2.81, p=0.012, d$ $=0.25,95 \% \mathrm{Cl}=[0.06,0.37]) ;$ however, this effect did not persist at 6 months. For psychedelic-naïve participants, larger effect sizes were detected at 4 weeks 
compared with baseline $(t(52)=3.38, p=0.003, d=0.46,95 \% \mathrm{Cl}=[0.16,0.63])$, and these changes persisted for at least 6 months $(t(52)=2.86, p=0.012, d=0.39$, $95 \% \mathrm{Cl}=[0.11,0.64])($ Supplementary Figure $2 \mathrm{a})$. Consistent with the results described above, correlational analysis revealed a mild $(r=0.186)$ but significant positive correlation $(p<0.0001)$ between baseline beliefs in Fatalistic Determinism and lifetime psychedelic-use (Supplementary Figure 2b and see Supplementary Figure 3 for correlations between scales at different timepoints).

\section{Conversion of preferred metaphysical beliefs}

To further explore the relationship between psychedelic use and shifts in participant's metaphysical positions, we separated the sample into 4 groups corresponding to which metaphysical position participants mostly strongly endorsed at baseline. Respondents with either no positive endorsement or scoring equally high on more than one item were grouped under the label 'none/mixed', otherwise they were categorised as either: dualists, idealists or materialists. Results showed baseline 'hard-materialists' tended to shift away from this position after psychedelic use. In fact, such shifts were more common than not. We also found that among those who did shift, the nature of this shift was either towards the 'none/mixed' position or 'hard-dualism'. Intriguingly, shifts away from polar metaphysical views was also evident for a large portion (37\%) of baseline 'hard-dualists' who tended to reject any preference or endorse an equanimous (i.e. mixed) position postpsychedelics (Figure 2a and Figure 2b). Separately, however, we observed that those who held more moderate views on 'panpsychism' became more convinced of this position post-psychedelics (labelled 'believers') (Figure 2c and Figure 2d). These 
prospective findings were matched by correlations between lifetime psychedelic use

and stronger panpsychist and weaker materialist views, at baseline. (Figure 2e).
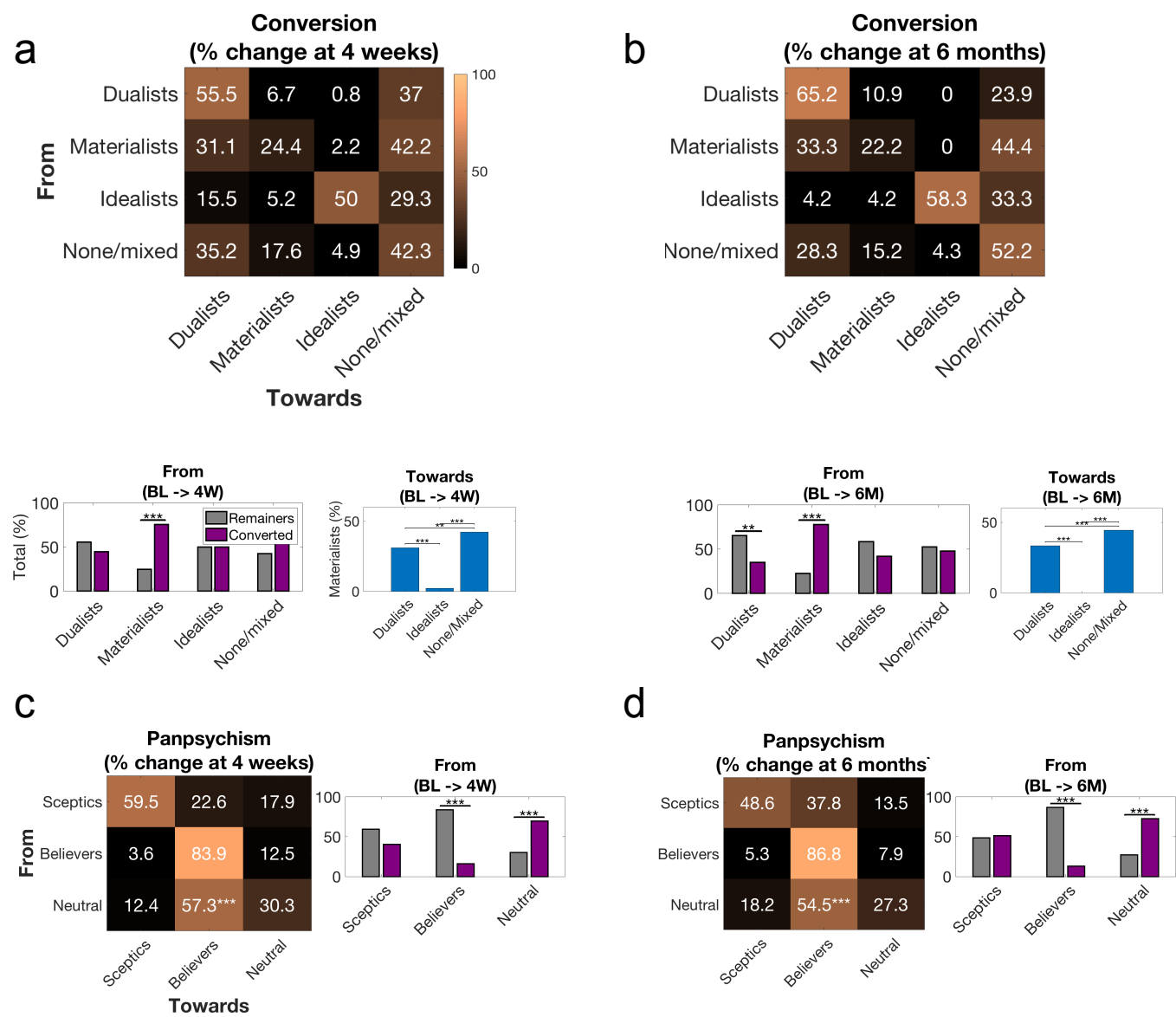

e

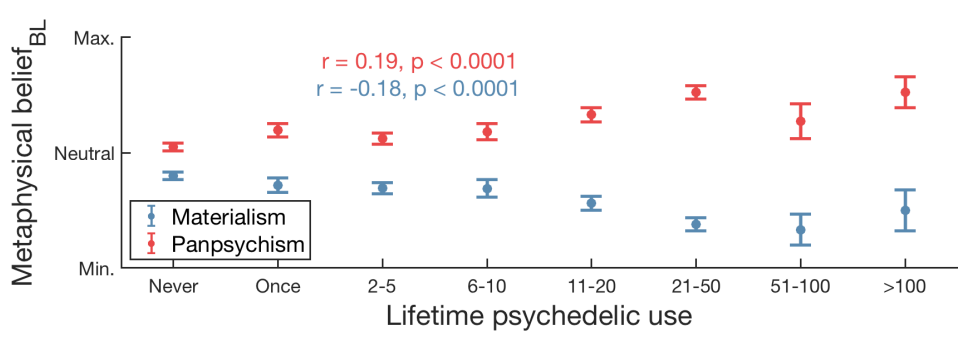

Figure 2. The nature of belief-shifts post psychedelic use. Matrices displaying the rate of belief-shift from and towards different 'hard' metaphysical positions are displayed at 4 weeks (a-above) and 6 months (b-above) following the retreat. Significant rates of change were found only for respondents' endorsing materialism at 4 weeks (a-below) and 6 months (bbelow), with most of these 'hard materialists' leaning towards dualism and equanimity (or reduced hard materialism) post-retreat. Significant rates of belief-shift were also found for respondents with non-committal views on panpsychism at baseline, who then shifted towards a panpsychist 'believer' stance at 4 weeks (c) and 6 months (d) post-retreat. (e) Lifetime psychedelic use was positively correlated with panpsychist views and negatively correlated with hard materialistic views measured at baseline. $\left({ }^{*} p<0.05,{ }^{* *} p<0.01\right.$, $\left.{ }^{* * *} p<0.001\right)$. 


\section{Non-physicalist Beliefs and well-being}

A significant positive correlation was found between shifts away from hardmaterialism (the NPB factor) and changes in well-being. The correlation was significant at 4 weeks and at 6 months post-retreat (Figure 3).

a

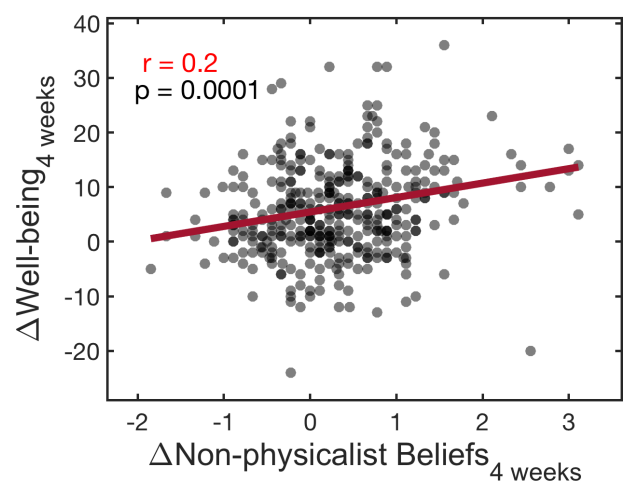

b

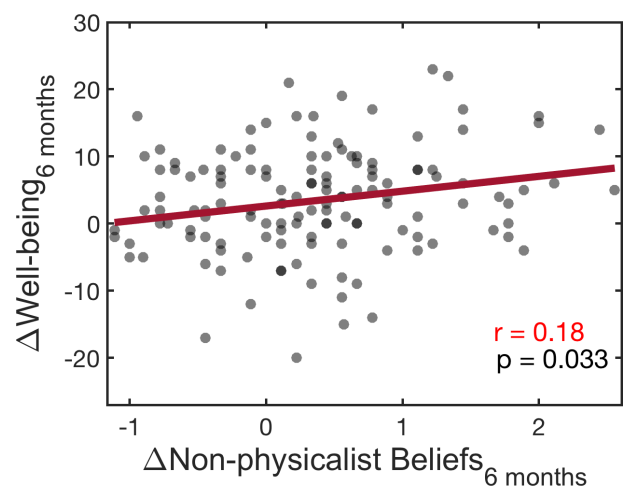

Figure 3. Shifts away from hard materialistic beliefs are associated with increases in well-being. A positive correlation was observed for shifts away from hard materialism versus changes in well-being at both (a) 4 weeks and (b) 6 months.

\section{Process of change modelling}

A path analysis was performed to examine mechanisms associated with shifts in the relevant non-physicalist beliefs (see Methods for details). Included in the model were items and scales pertaining to the acute subjective effects of psychedelics as well as environmental and social-contextual variables relevant to the retreat experience. Results supported a model with excellent fit (Supplementary Table 3) in which perceived 'emotional synchrony' with other participants - moderated by baseline scores of peer conformity - predicted subsequent changes in the NPB factor. Acute emotional synchrony was itself predicted by trait absorption, gender, 
age, baseline beliefs, plus identity fusion (i.e., identification with the retreat group)

assessed shortly prior to the experience (see Materials and Methods for details)

(Figure 4).

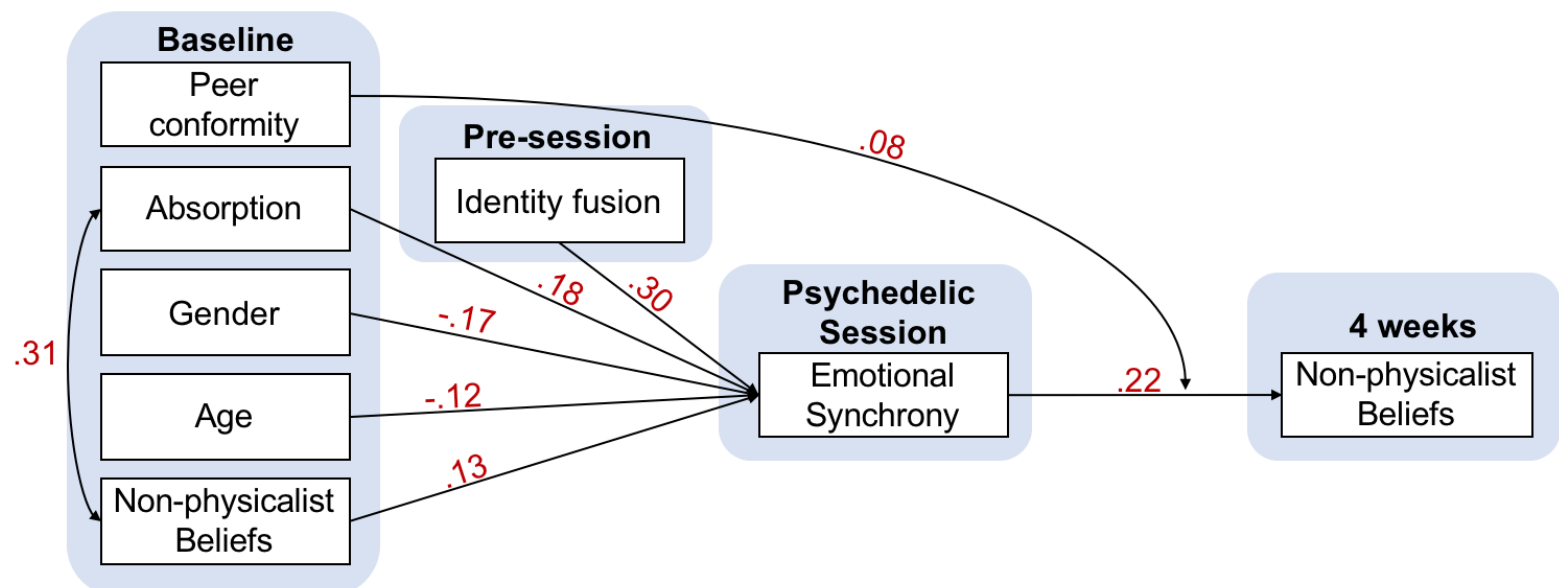

Figure 4. Changes in non-physicalist beliefs are moderated by baseline variables and pre-state identify fusion and mediated by acute emotional synchrony during the psychedelic session. Path model showing changes in Non-physicalist Beliefs to be affected by several demographic and trait characteristics including absorption, gender and age, mediated through perceived emotional synchrony during the psychedelic group session. The effect of synchrony on non-physicalist beliefs was conditional on respondents' baseline scores of peer conformity. Standardized $\beta$-coefficients are shown for significant $(p<$ .05) regression paths (not shown are additional significant correlations between nonphysicalist beliefs at baseline and absorption with gender, $r=.19$ and $r=.16$, respectively, as well as a significant effect between beliefs at baseline and at 4 weeks post-session; $\beta=$ .75 .

Validation with data from a controlled clinical trial

To test the validity and replicability of our findings, we administered items corresponding to the NPB during a double-blind randomized-controlled trial comparing a group $(n=30)$ receiving psilocybin therapy with another undergoing a 6week course of escitalopram ( $n=29$ ) (See Methods for details of trial design). Results replicated well across the independent studies. That is, a significant drug versus time (before treatment and 6 weeks after) interaction was observed $(F(56)=3.13, p=$ 
0.041, one-tailed). Post-hoc tests revel that shifts away from hard materialism were evident in the psilocybin group only $(Z=2.28, p=0.02, d=0.45)$. The escitalopram group showed no changes in NPB $(Z=0.24, p=0.33, d=0.2)$. (Figure 5a). Importantly, consistent with the above-reported findings of a relationship between belief shifts and positive mental health outcomes, significantly greater shifts away from hard materialistic beliefs (the NPB factor) were found for those patients who showed a clinically meaningful response (defined as at least $50 \%$ reduction in depression scores from baseline to week 6) only to psilocybin, as opposed to escitalopram $(Z=1.74, p=0.041, g=0.56,90 \% \mathrm{Cl}=[-0.17,1.26])$ (Figure 5b).

Finally, we found that the belief shifts in the psilocybin condition were largely correlated with positive endorsement of a unifying spiritual principle (see Supplementary Methods for the items used), indicating that changes in metaphysical beliefs are related to spiritual beliefs (Figure $5 c$ ). 

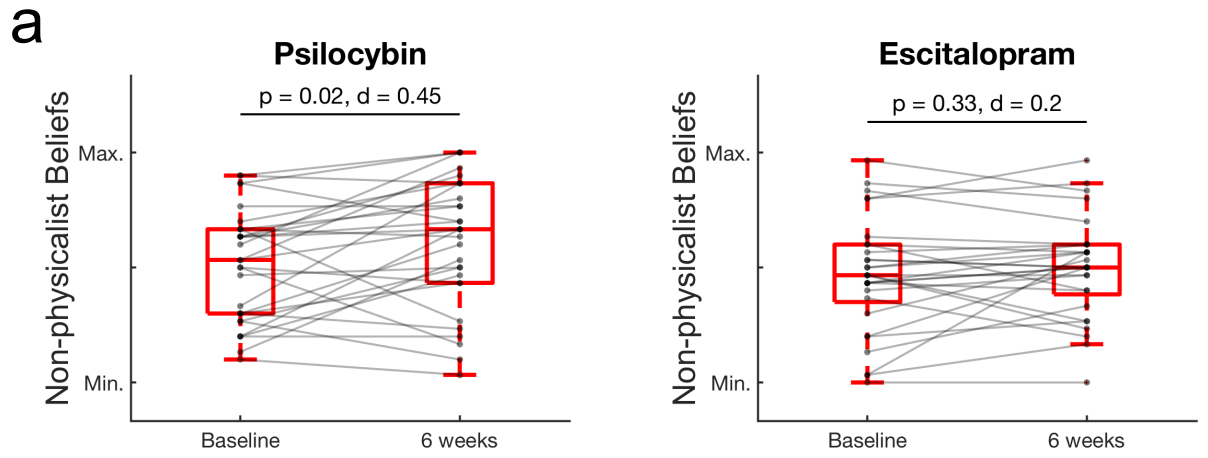

b

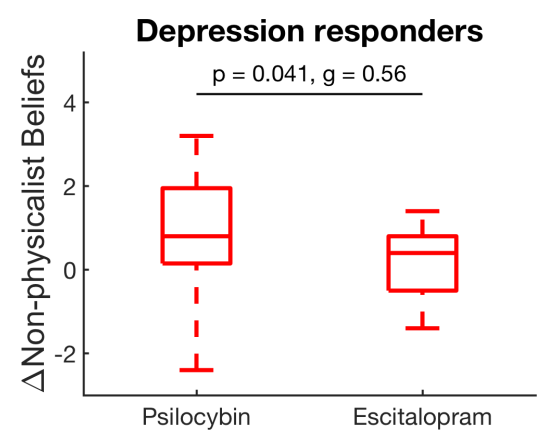

C

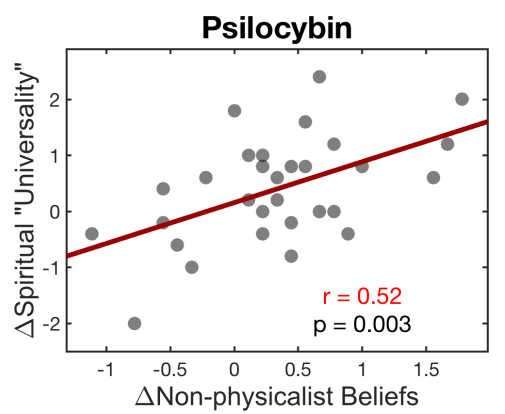

Figure 5. Consistent shifts away from physicalism after psilocybin therapy for depression: (a) Significant shifts away from hard physicalism were only seen for psilocybin and not the escitalopram control for the 6 weeks compared to baseline scores (Bonferronicorrected; $p$ values and Cohen's $d$ effect sizes shown). (b) Greater belief-shifts in the predicted direction were found for treatment responders in the psilocybin condition compared with the escitalopram groups ( $p$ value and Hedges' $g$ effect size shown). (c) Shift in non-physicalist beliefs were significantly associated with changes in spiritual domain 'Universality' 6 weeks minus baseline only for the psilocybin group.

\section{Discussion}

The present study sought to test the hypothesis that psychedelic experiences mediate changes in metaphysical beliefs concerning the nature of reality, consciousness and 'fate'. Converging cross-sectional, prospective observational and controlled research data suggest a relationship between psychedelic experiences and shifts away from positions of hard physicalism and towards panpsychism, dualistic, and fatalistic beliefs. The observed changes were enduring, persisting for up to 6 months in most domains. Moreover, the large-sample 
prospective/observational and smaller-sample but well-controlled research findings converged, implying that psychedelic-use may indeed be a casual determinant of the relevant shifts in metaphysical beliefs. Furthermore, the belief-shifts were correlated with positive mental health changes; namely, improvements in well-being in the observational data and depression scores in the controlled research data.

Path analyses on the psychedelic retreat-derived observational data highlighted the predictive relevance of certain psychological traits, including 'absorption' - which indexes differential proclivity to states of immersion, absorption, hyper-focus or flow $^{42}$, and peer conformity. Trait absorption has been found to predict propensity for spiritual-type experiences ${ }^{43}$ and be related to trait suggestibility ${ }^{42}$ - as well as a serotonin $2 \mathrm{~A}$ receptor genetic polymorphism ${ }^{44}$ - the key receptor target for classic psychedelics ${ }^{45}$. Peer conformity has also been found to relate to suggestibility ${ }^{46}$.

Regarding demographic variables, age and gender were other relevant predictors of belief shifts: specifically, lower age and female gender were predictive of the relevant changes. The relationship between lower age and suggestibility is well established ${ }^{47}$. Pre-state identity fusion (feelings of identification with the retreat group ${ }^{48}$ ) was another relevant variable moderating susceptibility to the relevant belief-shifts. All of these variables moderated 'emotional synchrony' 49 felt during the psychedelic ceremony itself. Higher emotional synchrony scores strongly mediated the relevant shifts in metaphysical beliefs, i.e., away from physicalism.

Taken together, these results imply an interaction between trait-level susceptibility to social influence and state-level group emotional alignment, 'attunement' or synchrony under the psychedelic, as a plausible mechanism for driving metaphysical belief-shifts. The role of differential susceptibility to environment bears relevance to 
popular models of individual differences in susceptibility to psychopathology, including those that make specific reference to genetic differences in serotonergic functioning ${ }^{50}$. Findings of increased (state) suggestibility under serotonergic psychedelics are interesting in this context ${ }^{51}$. The role of prior expectancy and acute suggestibility in moderating and mediating changes in mental health outcomes post psychedelic use is an area deserving more research ${ }^{52}$.

The present study's path modelling results may bear relevance to our understanding of the mechanisms underpinning other robust and often enduring psychological changes associated with psychedelic use, such as personality shifts ${ }^{53,54}$, improvements in mental health outcomes ${ }^{55}$, plus changes in ecological ${ }^{56,57}$, political ${ }^{58,59}$ spiritual $^{39}$ and religious positions ${ }^{29}$. These findings also add further weight to the principle that outcomes of psychedelic use strongly depend on contextual variables ${ }^{60}$. They also mesh well with work suggesting that ritual can enhance cultural transmission ${ }^{61}$, and it is logical to surmise that this effect could be further enhanced via the pro-suggestibility action of psychedelics. Advancing our understanding of the biological aspect of these psychological mechanisms associated with changes in beliefs is a research area deserving special focus. For example, the relationship between serotonin $2 \mathrm{~A}$ receptor agonism-induced cortical plasticity seems likely to be highly relevant in this regard ${ }^{15,62}$.

A recent predictive coding inspired model of the brain action of psychedelics, known as 'REBUS' (RElaxed Beliefs Under pSychedelics), may provide some useful inspiration for aiding investigations of the neurobiology of belief change processes ${ }^{63}$. The REBUS model proposes that rendering high-level beliefs and assumptions more plastic under psychedelics is a key mechanism underlying their acute 
phenomenological and potential therapeutic effects. Like previous work in other psychological domains (discussed above), our results indicate that changes in beliefs tend to occur in a particular direction (i.e., in the present case, away from physicalism). At the aggregate level, the dominant direction of this shift points away from 'hard' metaphysical views, and away from 'hard materialism' in particular. However, results also reveal signs of a 'hardening' (or increase in confidence) in beliefs in panpsychism and related domains of 'hard' dualism. More work is needed to address the question of whether the apparent directional nature of postpsychedelic belief-shifts are entirely context dependent, or whether there may also be a context-independent component to the changes. Indeed, it may transpire that these possibilities are not mutually exclusive, e.g., acute belief relaxation may be somewhat context independent but sub-acute belief revision is more dependent on context. This matter is related to the question of whether or not psychedelics are intrinsically 'healing'.

The role of contextual influences on post psychedelic belief-shifts, plus differential susceptibility to these, is an important topic to examine further, particularly given the present evidence indicating a significant role for social-psychological influences on key long-term outcomes. If, for example, the direction and nature of psychedelicinitiated belief changes depends entirely on contextual factors ${ }^{60,64,65}$ and differential susceptibility to these ${ }^{15,50}$, then this raises important bioethical implications, invoking, in the extreme, notions of 'mind-control' and 'brain-washing'66. These matters may be particularly relevant in relation to collective psychedelic experiences such as often occur at psychedelic retreats, in which the exchange of ideas, beliefs and worldviews are commonplace, and often centrally promoted ${ }^{40}$. However, note that belief-shifts in a consistent direction were also observed in the clinical trial data reported here, in 
which there was no conscious, intentional priming toward any particular metaphysical position.

Nonetheless, it is important to note that even in therapeutic and research environments, the psychedelic experience and surrounding process will be imbued with cultural factors that could easily influence beliefs in a particular direction ${ }^{40}$. Accounts of the apparent modulation of core beliefs via psychedelic drug use appears to be consistent across different Western cultures ${ }^{39}$, and the types of beliefs that participants appear to gravitate towards after psychedelic use are somewhat consistent with those that are culturally held by many indigenous and mestizo populations ${ }^{67,68}$, as well as some "New Age" groups ${ }^{69}$, particularly if they have connections with psychedelic drug use. One could interpret these observations as consistent with a context-independent belief shift, or alternatively, evidence of a bidirectional causal relationship between cultural values and psychedelic use, e.g., that certain cultures and viewpoints lend themselves toward psychedelic use, or even psychedelic research.

Assessing the value of competing philosophical positions is beyond the remit of the present work -, and, some might argue, beyond the remit of science. However, we acknowledge that this matter has important ethical, social and psychological implications and therefore merits some further comment. Thus, one pragmatic way in which science could approach this issue would be to assess how different metaphysical positions interface with individual (mental) health, as well as other indices of individual plus group or societal health, e.g., population-level well-being. The present study found a positive association between changes in metaphysical beliefs away from physicalism and increased psychological well-being. Moreover, 
this finding was replicated in independent data from controlled research where beliefshifts in a consistent direction were found in responders (vs. non-responders) to psilocybin-therapy for depression, and no such relationship was evident for an active-treatment (selective serotonin reuptake inhibitor, antidepressant, 'escitalopram') control arm, even in those who responded to that intervention.

It would be hasty, in our view, to interpret these findings as evidence in favour of the positive value of e.g., anti-materialist, pro-panpsychist or fatalistic beliefs. For example, the adoption of non-physicalist or supernatural beliefs have been associated with maladaptive coping strategies such as avoidance or escapism, e.g. via 'spiritual bypassing'70-73 . Furthermore, mistrust of scientifically-informed advice can foment problematic collective behaviours - such as a rejection of strategies for the prevention of COVID-19 transmission ${ }^{74}$. These issues may speak to the dangers of both an open-mindedness 'overshoot', and 'undershoot', with the former associated with paranoid, conspiratorial and escapist thinking and the latter, a potential cognitive fusion or rigidity linked to poor psychological well-being ${ }^{15,73}$. Again, however, these different presentations may not be mutually exclusive, e.g., some people may oscillate between them.

The principle that psychedelic experiences can mediate fundamental and enduring shifts in belief has important bioethical implications. One popular historical narrative is that spiralling psychedelic use in 1960s catalysed counter-cultural views and activities that provoked prohibitionist policies which effectively suspended research and clinical/therapeutic work with these compounds ${ }^{66}$. Recent evidence suggests that psychedelic use has increased exponentially in the last decade in the US $^{75}$ and is set to scale-up further due to increasing public interest ${ }^{76}$ and liberalising 
policies on access ${ }^{77}$. Greater research is therefore clearly needed on the societal implications of putative psychedelic-induced belief-shifts. See ${ }^{40,63,78}$ for relevant discussions.

A major advance of the present work is its synthesis of converging lines of evidence in support of a causal role for psychedelic use on the relevant belief changes. These lines of evidence include: 1) that the belief-shifts were observed prospectively (i.e. before vs after psychedelic use) in a large sample, 2) especially pronounced shifts were seen in psychedelic-naïve participants, 3) the quality of the acute experience (i.e., emotional synchrony) was found to be a significant mediator of the belief-shifts, 4) extent of past psychedelic use correlated with beliefs in the predicted direction, and 5) independent data from a separate controlled study in a distinct population, replicated findings from our observational data. It is also relevant that previous controlled studies have demonstrated post-psychedelic changes in other psychological domains after controlling for potential confounding factors $29,56,57$.

To conclude, this mixed method study, comprising of an observational plus controlled research design, has comprehensively assessed the relationship between psychedelic drug use and metaphysical beliefs. Findings converged on the inference that psychedelic use inclines individuals away from hard physicalist beliefs and towards dualistic, panpsychist, and fatalistic beliefs, thus highlighting the potential of psychedelics to alter some of the most deep-seated and influential human beliefs. These results have profound scientific, societal, political and philosophical implications, and therefore demand further investigation. 


\section{Methods}

\section{Design and Participants}

Respondents planning to attend a ceremony involving a psychedelic substance (psilocybin/magic mushrooms/truffles, ayahuasca, DMT, San Pedro, LSD/1P-LSD) were recruited via online advertisements and invited to sign up for the study via the website www.psychedelicsurvey.com. Eligibility criteria to participate consisted in: being 18 years or older, good comprehension of the English language, and the intention to participate in a retreat, ceremony or other guided experience involving a classic psychedelic (i.e., 5-HT2A agonist such as psilocybin, DMT, mescaline or LSD). The study received a favourable opinion from the Imperial College Research Ethics Committee and was sponsored by the Imperial Joint Research and Compliance Office. All participants provided informed consent. Automatic email reminders were then sent out at multiple time-points at baseline (one week before the experience), one day after the experience and 4 weeks after the indicated date of the experience to participants who signalled consent, including links to surveys hosted on the online platform SurveyGizmo. All methods were carried out by relevant guidelines and regulations (see Kettner et al. ${ }^{79}$ for details).

Survey findings were complemented by results from controlled a controlled clinical-trial comparing psilocybin versus escitalopram treatment for major depression. The data from 59 participants with major depression (>17 on HamiltonDepression [HAM-D-17] scale at screening) who completed the trial was used (30 participants for psilocybin and 29 for escitalopram). Participants ranging from 18 - 80 years old were recruited formally through trial networks, informally via social media, and through other sources. Main exclusion criteria consisted in immediate family or 
personal history of psychosis, medically significant health conditions (assessed by a physician), a history of serious suicide attempts, positive pregnancy test, contraindications to undergo an MRI or taking selective serotonin-reuptake inhibitors (SSRI's), previous use of escitalopram or a pre-existing condition that could jeopardize the rapport between the participant and the trial mental health caregivers. Participants received either 2 doses of an active dose of psilocybin $(25 \mathrm{mg})$ and 6 weeks of daily placebo (psilocybin group) or 2 doses of a control dose of psilocybin (1 mg) and daily escitalopram (escitalopram group). Participants completed an adapted version of the Metaphysical Beliefs Questionnaire (see Measures) at baseline and at 6 weeks following the start of treatment, the latter being the key endpoint of the trial. Home Office Schedule 1 Drug Licenses, UK Medicines \& Healthcare products Regulatory Agency, and GDPR approvals were obtained. All study protocols were approved by the Brent Research Ethics Committee, the Health Research Authority, Imperial College London and the Joint Research Compliance office. All methods were carried out by relevant guidelines and regulations (see Carhart-Harris et al. ${ }^{80}$ for details regarding the study protocol and the main results of the trial). (ClinicalTrials.gov Identifier: NCT03429075, registered on February 12, 2018; EudraCT: 2017-000219-18).

\section{Measures}

There were two main outcome measures in this study which were employed at baseline (one week prior to the experience), 4 weeks, and 6 months after attending the retreat. They were (1) a self-constructed Metaphysical Beliefs Questionnaire (MBQ; see Supplementary Table 2), and (2) items extracted from the Free Will and Fatalistic Determinism subscales (containing 9 items in total) of the FAD-Plus 
questionnaire, a validated measure of lay views on free will and determinism ${ }^{4}$ (see Supplementary Methods for the items used).

The MBQ consists of 13 items: 3 directly assessing beliefs concerning the mental and the physical (i.e. beliefs in physicalism/materialism, idealism and dualism) - that were directly extracted from previous work $^{6}$, and 10 that were conceived based on a review of literature pertaining to psychedelic-induced changes in metaphysical beliefs $^{23-25,81}$, qualitative studies ${ }^{82-84}$, and transcribed interviews performed in our own research involving DMT and psilocybin administrations to healthy volunteers 85,86 . The relevant 10 items were conceived with the intention of assessing beliefs in separate domains, including panpsychism, idealistic/solipsistic beliefs (i.e. 'the physical world is an illusion generated by the mind'), beliefs in the literal transcendence of the constraints of this universe, and related beliefs in the existence of other worlds or universes that one can visit, monism ( i.e. the position that there is only one fundamental type of substance), idealism (i.e. that reality is entirely constituted by mind or consciousness), physicalism or materialism (i.e. the belief that reality is entirely constituted by physical or material objects and processes), the notion that all mental events (including the sense of self) can be traced back to brain activity and more nuanced positions highlighting the embeddedness of conscious experience in brain, body and world (i.e. enactivism ${ }^{87}$ ).

Additionally, we explored the acute experiences of ceremony participants by issuing questionnaires enquiring about this one day after the experience itself. The Mystical Experience Questionnaire (MEQ; ${ }^{21}$ ) and Perceived Emotional Synchrony Scale $\left(\mathrm{PESC} ;{ }^{88}\right)$ were measured in relation to the acute psychedelic experience. Additionally, the personality trait Absorption ${ }^{42}$ (known to be a contextual factor which 
can strongly predict the character of psychedelic experiences ${ }^{89}$ ) and the subscale peer conformity from the Multidimensional lowa Suggestibility Scale ${ }^{46}$ (suggestibility is known to be sensitive to psychedelic administration ${ }^{51}$ ) were both measured at baseline. Finally, identity fusion (the visceral experience of 'oneness' with the group) was measured shortly before the session as a single pictographic item ${ }^{48}$.

We also explored the extent to which any changes in beliefs might be associated with changes in psychological well-being. Well-being was measured at baseline, 4 weeks after, and 6 months after the ceremony using the Warwick-Edinburgh Mental Wellbeing Scale ${ }^{90}$, which has been shown to be sensitive to the effects of psychedelics $56,91,92$.

For controlled research, a shortened version of the Metaphysical Beliefs Questionnaire was used (containing the following items: Ontological Transcendentalism, Supernatural Transcendentalism, Dualism, Materialism, and Non-naturalism), from which the NPB factor was extracted. Also, for the controlled study, the 'Universality' subscale from the Spiritual Transcendence Scale ${ }^{93}$ (see Supplementary Methods for the items) was used to determine the association between changes in beliefs and changes in spirituality.

\section{Statistical Analysis}

An exploratory factor analysis was performed on the Metaphysical Beliefs Questionnaire (see Supplementary Table 2 for all items) at baseline. Multiple heuristics were used to determine the optimal number of factors to retain among the 13 items included in the analysis ${ }^{94}$. Parallel analysis, optimal coordinate and the 'Kaiser rule'95, suggested a three-factorial solution, whereas acceleration factor and 
visual examination of the scree plot a unifactorial solution (Supplementary Figure 4). Examination of factor loadings showed that in the three-factorial solution, only one and two items loaded on factors two and three, respectively. Thus, a unifactorial solution was chosen, with standardized loadings presented in table 1. After removal of items with a. factor loading <.4 ${ }^{96}$. Finally, Cronbach's alpha was used to determine internal consistency.

Analyses were conducted to determine changes in beliefs (baseline vs 4 week post ceremony, and baseline vs 6 months post ceremony) for all respondents. Separate analyses were performed using responses from participants with no previous psychedelic experiences, on the grounds that respondents with previous psychedelic experiences might already have had shifts in the relevant beliefs. Paired t-tests were performed to assess the statistical significance of changes in specific beliefs (the principal factor extracted from the $M B Q$ and the Free-Will and Fatalistic Determinism subscales from the FAD scale). Two-tailed and Bonferroni-corrected pvalues are reported. Cohen's $d$ effect sizes are reported for all paired analyses.

Following previous studies ${ }^{6}$, we also performed analyses to determine the rate of conversion from baseline 'hard' metaphysical positions regarding materialism, idealism and dualism. In order to do this, we divided the groups based on their tendency to endorse materialism, dualism, idealism or 'none/mixed' based on their initial Likert responses for items 3, 4 and 5 of the MBQ (see Supplementary Table 2). The single highest score between the 3 items corresponded to the group each respondent would be allocated. If there were competing highest scores, or if the highest score was below or equal to a neutral score, then subjects were grouped as Agnostics. Determining the rate of conversion from a hard metaphysical position was 
done via Chi-squared tests comparing percentages of 'Remainers' (those respondents who did not show any change according in groups for subsequent timepoints) and 'Converted' (those who did in any direction). Determining differences between the groups toward which Materialists respondents converted (the only group showing a significant rate of conversion) was also done by performing paired Chi-squared tests for each pair of comparisons ( $p$ values were Bonferroni-corrected for multiple comparisons). We also performed a similar procedure using a single item corresponding to panpsychism from the MBQ, by classifying subjects as 'Believers', 'Sceptics' and 'Neutral' according to scores above, below or corresponding to, the neutral Likert score. Chi-Squared tests were performed for comparing Conversion from and towards a different metaphysical position.

A longitudinal exploratory path analysis was performed to determine the psychological mechanisms associated with changes in NPB. Included were items and scales pertaining to the acute subjective effects of the psychedelic as well as environmental and social contextual variables relevant to the retreat experience. Specifically, the intensity of mystical-type (MEQ) and emotional synchrony experiences (PESC), measured on the day post-session, were expected to predict baseline-corrected NPB scores 4 weeks post-session, although due to nonsignificance of effects, only the PESC was retained in the model. In the case of collective emotional synchrony experiences, this effect was expected to be moderated by peer conformity measured at baseline, calculated as the sum of the 4 peer conformity-items from the short suggestibility scale (SSS). Demographic variables, trait absorption (MODTAS), baseline supernatural NPB, and alignment with the group assessed hours before the session via a pictographic identity fusion scale ${ }^{48}$ were included in the model as predictors of acute experience (MEQ and 
PESC) scores. Whenever a respondent attended more than one psychedelic ceremony and provided multiple reports, only the largest score for each of these scales was considered.

The association between changes in NPB and mental health outcomes was assessed by Pearson-Point correlational analyses between changes in the NPB factor and changes in wellbeing (changes in WEBMWS scores).

For the controlled research analysis, changes in NPB between baseline and the 6-weeks timepoint was compared between the psilocybin and escitalopram groups using a repeated measures ANCOVA, adjusted for baseline. Post-hoc tests comparing baseline and 6-weeks post-treatment were performed using Wilcoxon signed rank test for dependent samples (Bonferroni-corrected). Changes in NPB Beliefs were compared for psilocybin versus escitalopram remitters using Wilcoxon rank sum test for independent samples. Finally, the association between Changes in NPB and changes in Spiritual Beliefs were assessed using the Universality subscale from the Spiritual Transcendence Scale ${ }^{93}$. Single-sided $p$-values are reported for controlled research analysis as clear hypotheses were derived from the main survey data presented here. Cohen's $d$ and Hedges' $g$ effect sizes are reported for paired and independent tests, respectively. 


\section{References}

1. van Inwagen, P. \& Sullivan, M. Metaphysics. The Stanford Encyclopedia of Philosophy (2018). Available at:

https://plato.stanford.edu/archives/spr2018/entries/metaphysics/. (Accessed: 4th January 2020)

2. Forstmann, M. \& Burgmer, P. Adults are intuitive mind-body dualists. J. Exp. Psychol. Gen. 144, 222-235 (2014).

3. Stanovich, K. E. Implicit philosophies of mind: the dualism scale and its relation to religiosity and belief in extrasensory perception. J. Psychol. 123, 523 (1989).

4. Paulhus, D. L. \& Carey, J. M. The FAD-plus: Measuring lay beliefs regarding free will and related constructs. J. Pers. Assess. 93, 96-104 (2011).

5. Demertzi, A. et al. Dualism Persists in the Science of Mind. Ann. New York Acad. Sci. 1157, 1-9 (2009).

6. Reggia, J. A., Huang, D.-W. \& Katz, G. Beliefs concerning the nature of consciousness. J. Conscious. Stud. 146-171 (2015).

7. Bering, J. M. The folk psychology of souls. Behav. Brain Sci. 29, 453-498 (2006).

8. Forstmann, M., Burgmer, P. \& Mussweiler, T. "The mind is willing, but the flesh Is weak": The effects of mind-body dualism on health behavior. Psychol. Sci. 23, $1239-1245$ (2012).

9. Vohs, K. D. \& Schooler, J. W. The value of believing in free will: Encouraging a belief in determinism increases cheating. Psychol. Sci. 19, 49-54 (2008).

10. Baumeister, R. F., Masicampo, E. J. \& Dewall, C. N. Prosocial benefits of feeling free: Disbelief in free will increases aggression and reduces 
helpfulness. Personal. Soc. Psychol. Bull. 35, 260-268 (2009).

11. Aarts, H. \& van den Bos, K. On the foundations of beliefs in free will:

Intentional binding and unconscious priming in self-agency. Psychol. Sci. 22, 532-537 (2011).

12. O'Connor, T. \& Franklin, C. Free Will. The Stanford Encyclopedia of Philosophy (2019).

13. Flanagan, O. \& Caruso, G. D. Neuroexistentialism. 1, (Oxford University Press, 2018)

14. Miller, W. R. The Phenomenon of Quantum Change. J. Clin. Psychol. 60, 453$460(2004)$

15. Brouwer, A. \& Carhart-Harris, R. L. Pivotal Mental States. Journal of Psychopharmacology (2020). doi:10.1177/0269881120959637

16. Greyson, B. The near-death experience scale. Construction, reliability, and validity. The Journal of nervous and mental disease 171, 369-375 (1983).

17. Josipovic, Z. Duality and nonduality in meditation research. Conscious. Cogn. 19, 1119-1121 (2010).

18. Lynn, S. J. \& Evans, J. Hypnotic suggestion produces mystical-type experiences in the laboratory: A demonstration proof. Psychol. Conscious. Theory, Res. Pract. 4, 23-37 (2017).

19. White, F. The overview effect: Space exploration and human evolution. (Houghton Mifflin, 1987).

20. Edmondson, D. et al. From shattered assumptions to weakened worldviews: Trauma symptoms signal anxiety buffer disruption. J. Loss Trauma 16, 358$385(2011)$

21. Barrett, F. S., Johnson, M. W. \& Griffiths, R. R. Validation of the revised 
Mystical Experience Questionnaire in experimental sessions with psilocybin. J. Psychopharmacol. 0269881115609019- (2015).

doi:10.1177/0269881115609019

22. Pahnke, W. N. \& Richards, W. A. Implications of LSD and experimental mysticism. J. Psychoactive Drugs 3, 92-108 (1970).

23. Grof, S. Realms of the Human Unconscious. (Viking, 1975).

24. Strassman, R. DMT: The spirit molecule. (Park Street Press, 2001).

25. Shanon, B. The Antipodes of the Mind: Charting the Phenomenology of the Ayahuasca Experience. (Oxford University Press, 2005).

26. Davis, A. K. et al. Survey of entity encounter experiences occasioned by inhaled N,N -dimethyltryptamine: Phenomenology, interpretation, and enduring effects. J. Psychopharmacol. 026988112091614 (2020).

doi:10.1177/0269881120916143

27. Griffiths, R. R., Richards, W. A., Johnson, M. W., McCann, U. D. \& Jesse, R. Mystical-type experiences occasioned by psilocybin mediate the attribution of personal meaning and spiritual significance 14 months later. $J$.

Psychopharmacol. 22, 621-632 (2008).

28. Kometer, M., Pokorny, T., Seifritz, E. \& Volleinweider, F. X. Psilocybin-induced spiritual experiences and insightfulness are associated with synchronization of neuronal oscillations. Psychopharmacology (Berl). 232, 3663-3676 (2015).

29. Griffiths, R. R., Hurwitz, E. S., Davis, A. K., Johnson, M. W. \& Jesse, R. Survey of subjective 'God encounter experiences': Comparisons among naturally occurring experiences and those occasioned by the classic psychedelics psilocybin, LSD, ayahuasca, or DMT. PLoS One 14, 1-26 (2019). 
30. Ross, S. et al. Rapid and sustained symptom reduction following psilocybin treatment for anxiety and depression in patients with life-threatening cancer: a randomized controlled trial. J. Psychopharmacol. 30, 1165-1180 (2016).

31. Forstmann, M., Yudkin, D. A., Prosser, A. M. B., Heller, S. M. \& Crockett, M. J. Transformative experience and social connectedness mediate the moodenhancing effects of psychedelic use in naturalistic settings. Proc. Natl. Acad. Sci. 1-9 (2020). doi:10.1073/pnas.1918477117

32. Maslow, A. H. Cognition of being in the peak experiences. J. Genet. Psychol. 94, 43-66 (1959).

33. Stace, W. Mysticism and Philosophy. (J. B. Lippincott, 1960).

34. Richards, W. A., Rhead, J. C., DiLeo, F. B., Yensen, R. \& Kurland, A. A. The Peak Experience Variable In DPT-Assisted Psychotherapy with Cancer Patients. J. Psychedelic Drugs 9, 1-10 (1977).

35. Shiota, M. N., Keltner, D. \& Mossman, A. The nature of awe: Elicitors, appraisals, and effects on self-concept. Cogn. Emot. 21, 944-963 (2007).

36. Yaden, D. B. et al. The overview effect: Awe and self-transcendent experience in space flight. Psychol. Conscious. Theory, Res. Pract. 3, 1-11 (2016).

37. Hanley, A. W., Nakamura, Y. \& Garland, E. L. The Nondual Awareness Dimensional Assessment (NADA): New tools to assess nondual traits and states of consciousness occurring within and beyond the context of meditation. Psychol. Assess. 30, 1625-1639 (2018).

38. Miller, W. R. \& C'Dde Baca, J. Quantum Change: When Epiphanies and Sudden Insights Transform Ordinary Lives. (Guilford Press, 2001).

39. Lerner, M. \& Lyvers, M. Values and beliefs of psychedelic drug users: A crosscultural study. J. Psychoactive Drugs 38, 143-147 (2006). 
40. Timmermann, C., Watts, R. \& Dupuis, D. Towards psychedelic apprenticeship: Developing a gentle touch for the mediation and validation of psychedelicinduced insights and revelations. PsyArxiv 1-29 (2020). doi:10.31234/osf.io/j5768

41. Johnson, M. W. \& Yaden, D. B. There's no good evidence that psychedelics can change your politics or religion. Scientific American (2020). Available at: https://www.scientificamerican.com/article/theres-no-good-evidence-thatpsychedelics-can-change-your-politics-or-religion/.

42. Jamieson, G. A. The modified Tellegen Absorption Scale: A clearer window on the structure and meaning of absorption. Aust. J. Clin. Exp. Hypn. 33, 119-139 (2005).

43. Lifshitz, M., van Elk, M. \& Luhrmann, T. M. Absorption and spiritual experience: A review of evidence and potential mechanisms. Conscious. Cogn. 73, 102760 (2019).

44. Ott, U., Reuter, M., Hennig, J. \& Vaitl, D. Evidence for a common biological basis of the absorption trait, hallucinogen effects, and positive symptoms: Epistasis between 5-HT2a and COMT polymorphisms. Am. J. Med. Genet. Neuropsychiatr. Genet. 137 B, 29-32 (2005).

45. Nichols, D. E. Psychedelics. Pharmacol. Rev. 68, 264-355 (2016).

46. Kotov, R., Bellman, S. B. \& Watson, D. B. Multidimensional lowa suggestibility scale (MISS) bried manual. Soneybrook Med. Website (2004).

47. Mitchell, K. J., Johnson, M. K. \& Mather, M. Source monitoring and suggestibility to misinformation: Adult age-related differences. Appl. Cogn. Psychol. 17, 107-119 (2003).

48. Swann, W. B., Gómez, Á., Seyle, D. C., Morales, J. F. \& Huici, C. Identity 
Fusion: The Interplay of Personal and Social Identities in Extreme Group

Behavior. J. Pers. Soc. Psychol. 96, 995-1011 (2009).

49. Páez, D., Rimé, B., Basabe, N., Wlodarczyk, A. \& Zumeta, L. Psychosocial effects of perceived emotional synchrony in collective gatherings. J. Pers. Soc. Psychol. 108, 711-729 (2015).

50. Van ljzendoorn, M. H., Belsky, J. \& Bakermans-Kranenburg, M. J. Serotonin transporter genotype 5HTTLPR as a marker of differential susceptibility A meta-analysis of child and adolescent gene-by-environment studies. Transl. Psychiatry 2, (2012).

51. Carhart-Harris, R. L. et al. LSD enhances suggestibility in healthy volunteers. Psychopharmacology (Berl). 232, 785-794 (2015).

52. Kaertner, L. S. et al. Positive expectations predict improved mental-health outcomes linked to psychedelic microdosing. Sci. Rep. 11, 1-11 (2021).

53. Erritzoe, D. et al. Effects of psilocybin therapy on personality structure. Acta Psychiatr. Scand. 138, 368-378 (2018).

54. MacLean, K. A., Johnson, M. W. \& Griffiths, R. R. Mystical experiences occasioned by the hallucinogen psilocybin lead to increases in the personality domain of openness. J. Psychopharmacol. 25, 1453-1461 (2011).

55. Carhart-Harris, R. L. \& Goodwin, G. M. The Therapeutic Potential of Psychedelic Drugs: Past, Present and Future. Neuropsychopharmacology 1-9 (2017). doi:10.1038/npp.2017.84

56. Kettner, H., Gandy, S., Haijen, E. C. H. M. \& Harris, R. L. C. From egoism to ecoism: Psychedelics increase nature relatedness in a state - mediated and context - dependent manner. J. Environ. Res. Public Heal. 16, 1-25 (2019).

57. Forstmann, M. \& Sagioglou, C. Lifetime experience with (classic) psychedelics 
predicts pro-environmental behavior through an increase in nature relatedness. J. Psychopharmacol. 31, 975-988 (2017).

58. Nour, M. M., Evans, L. \& Carhart-Harris, R. L. Psychedelics, Personality and Political Perspectives. J. Psychoactive Drugs 49, 182-191 (2017).

59. Lyons, T. \& Carhart-Harris, R. L. Increased nature relatedness and decreased authoritarian political views after psilocybin for treatment-resistant depression.

J. Psychopharmacol. 026988111774890 (2018).

doi:10.1177/0269881117748902

60. Carhart-Harris, R. et al. Psychedelics and the essential importance of context. J. Psychopharmacol. 32, 725-731 (2018).

61. Whitehouse, H. Modes of religiosity: A cognitive theory of religious transmission. (AltaMira Press, 2004).

62. Ly, C. et al. Psychedelics Promote Structural and Functional Neural Plasticity. CellReports 1-13 (2018). doi:10.1016/j.celrep.2018.05.022

63. Carhart-Harris, R. L. \& Friston, K. J. REBUS and the anarchic brain: Toward a unified model of the brain action of psychedelics. Pharmacol. Rev. 71, 316344 (2019).

64. Hartogsohn, I. Set and setting, psychedelics and the placebo response: An extra-pharmacological perspective on psychopharmacology. J. Psychopharmacol. 30, 1259-1267 (2016).

65. Dupuis, D. The socialization of hallucinations. Cultural priors, social interactions and contextual factors in the use of ayahuasca. Transcult. Psychiatry (2020).

66. Lee, M. \& Shlain, B. Acid Dreams: The Complete Social History of LSD. (Grove Press, 1985). 
67. Labate, B. Ayahuasca Shamanism in the Amazon and Beyond. Ayahuasca

Shamanism in the Amazon and Beyond (2014).

doi:10.1093/acprof:oso/9780199341191.001.0001

68. Luna, L. E. The concept of plants as teachers among four mestizo shamans of iquitos, Northeastern Peru. J. Ethnopharmacol. 11, 135-156 (1984).

69. Hanegraaf, W. J. New Age religion and Western culture. Esotericism in the mirror of secular thought. (University of Utrecht, 1995).

70. Welwood, J. Toward a psychology of spiritual awakening. (Shambhala, 2002).

71. Masters, R. A. Spiritual Bypassing. (North Atlantic Books, 2010).

72. Kornfield, J. After the ecstasy, the laundry. (Rider, 2000).

73. Hayes, S. C. A liberated mind. (Avery, 2019).

74. Eaton, L. A. \& Kalichman, S. C. Social and behavioral health responses to COVID-19: lessons learned from four decades of an HIV pandemic. J. Behav. Med. 43, 341-345 (2020).

75. Yockey, R. A., Vidourek, R. A. \& King, K. A. Trends in LSD use among US adults: 2015-2018. Drug Alcohol Depend. 212, 2015-2018 (2020).

76. Pollan, M. How To Change Your Mind. (Penguin Press, 2018).

77. Naftulin, J. Oregon has become the first state to legalize 'magic' mushrooms for therapeutic use. Here's what that means. (2020). Available at: https://www.businessinsider.com/oregon-first-ever-state-to-legalize-psilocybinfor-therapeutic-use-2020-11?r=US\&IR=T. (Accessed: 12th November 2020)

78. Letheby, C. The epistemic innocence of psychedelic states. Conscious. Cogn. 39, 28-37 (2016).

79. Kettner, H. et al. Psychedelic Communitas: Intersubjective Experience During Psychedelic Group Sessions Predicts Enduring Changes in Psychological 
Wellbeing and Social Connectedness. Front. Pharmacol. 12, 1-20 (2021).

80. Carhart-Harris, R. et al. Trial of Psilocybin versus Escitalopram for Depression. N. Engl. J. Med. 384, 1402-1411 (2021).

81. Shanon, B. The epistemics of ayahuasca visions. Phenomenol. Cogn. Sci. 9, 263-280 (2010).

82. Watts, R., Day, C., Krzanowski, J., Nutt, D. \& Carhart-Harris, R. Patients' Accounts of Increased "Connectedness" and "Acceptance" After Psilocybin for Treatment-Resistant Depression. J. Humanist. Psychol. 57, 520-564 (2017).

83. Swift, T. C. et al. Cancer at the Dinner Table: Experiences of Psychotherapy for the Treatment of Cancer-Related Distress. J. Humanist. Psychol. 57, 488519 (2017).

84. Gasser, P., Kirchner, K. \& Passie, T. LSD-assisted psychotherapy for anxiety associated with a life-threatening disease: A qualitative study of acute and sustained subjective effects. J. Psychopharmacol. 29, 57-68 (2015).

85. Timmermann, C. et al. DMT models the near-death experience. Front. Psychol. 9, 1-12 (2018).

86. Turton, S., Nutt, D. J. \& Carhart-Harris, R. L. A qualitative report on the subjective experience of intravenous psilocybin administered in an fMRI environment. Curr. Drug Abuse Rev. 7, 117-127 (2014).

87. Varela, F. J., Thompson, E. \& Rosch, E. The Embodied Mind: Cognitive Science and Human Experience. The embodied mind Cognitive science and human experience (1991).

88. Wlodarczyk, A. et al. Perceived Emotional Synchrony in Collective Gatherings: Validation of a Short Scale and Proposition of an Integrative Measure. Front. Psychol. 11, 1-13 (2020). 
89. Studerus, E., Gamma, A., Kometer, M. \& Vollenweider, F. X. Prediction of psilocybin response in healthy volunteers. PLoS One 7, (2012).

90. Tennant, R. et al. The Warwick-Edinburgh mental well-being scale (WEMWBS): Development and UK validation. Health Qual. Life Outcomes 5, $1-13$ (2007).

91. Carhart-Harris, R. L. et al. Psilocybin with psychological support for treatmentresistant depression: an open-label feasibility study. The Lancet Psychiatry 3, 619-627 (2016).

92. Haijen, E. C. H. M. et al. Predicting responses to psychedelics : A prospective study. 9, 1-20 (2018).

93. Piedmont, R. L. Spiritual transcendence and the scientific study of spirituality. J. Rehabil. 67, 4-14 (2001).

94. Raîche, G., Walls, T. A., Magis, D., Riopel, M. \& Blais, J. G. Non-graphical solutions for Cattell's scree test. Methodology 9, 23-29 (2013).

95. Kaiser, H. F. The application of electronic computers to factor analysis. Educ. Psychol. Meas. XX, 141-151 (1960).

96. Guadagnoli, E. \& Velicer, W. F. Relation of Sample Size to the Stability of Component Patterns. Psychol. Bull. 103, 265-275 (1988). 


\section{Author contributions}

C.T., H.K., C.L., and R.L.C-H. designed the research. H.K. collected the data. C.T. and H.K. analysed the data, L.R. and F.E.R. provided analysis tools. C.T. and R.L.C-H. wrote the manuscript. All authors edited the manuscript.

\section{Acknowledgements}

This research was funded by the Ad Astra Chandaria Foundation, Alexander Mosley Charitable Trust and the funders of the Centre for Psychedelic Research (www.imperial.ac.uk/psychedelic-research-centre/ funding-partners/). Dr Letheby's contribution to this research was partially supported by the Australian Government through the Australian Research Council's Discovery Projects funding scheme (project DP190101451). The views expressed herein are those of the authors and are not necessarily those of the Australian Government or Australian Research Council.

\section{Additional information}

No competing interests declared. 